\title{
Cytokines and immune response in vasculitis of peripheral nervous system: Tumor necrosis factor-alpha and pro- inflammatory cytokines in vaculitis of peripheral nervous system: A comparative immunohistochemical study on systemic and non-systemic vasculitic neuropathy
}

\author{
Giovanni Antioco Putzu* \\ Casa di Cura Polispecialistica Sant'elena, Italy
}

\begin{abstract}
Objectives: To possibly differentiate immune response of SVN and NSVN by immunohistochemistry in sural nerve biopsies and to further characterize immune response in vasculitis of paraneoplastic syndrome.

Patients and methods: We performed an immunohistochemical study with a panel of antibodies directed against TNF-alpha, INF-gamma, IL-1Beta, IL-4, CD68, CD11, CD3, CD21, ICAM-1 and C5b9 in the peripheral nerve of seventy-five patients affected by vasculitis. Sixty patients (33 males and 27 females, mean age 63 years) were classified as SVN whereas 15 patients ( 7 males and 8 females, mean age 52 years) had NSVN.

Results: Results suggested a prevalent Th1 immune response in both SVN e NSVN. The presence of great amounts of TNF alpha and IL-1Beta into the vasculature determines a pro-coagulant effect with thrombosis of the vasa nervorum and hypoxia of the nervous fascicules. TNF-alpha was localized in axons as well, suggesting that it may be responsible for axonal degeneration per se. Peripheral nerves of SNV e NSVN were immunoreactive to INF-gamma antibodies. This finding indirectly confirms the presence in the vasculature of IL-28A, a powerful pro-inflammatory cytokine. CD11, an antibody reacting with leucocytes and ICAM-1 (Intercellular Adhesion Molecule-1) was also detected in the peripheral nerve of SVN and NSVN. Immunoreactivity to CD68 (macrophage antigen) and C5b9 (activated complement fraction) were also easily detected.

Conclusions: Results suggest that immune response in SVN and NSVN is of Th-1 type (cellular) in both SVN and NSVN. The role of TNF-alpha and cytokines is crucial in vascular thrombosis. Axonal degeneration may be the consequence of both hypoxia and direct toxic effect of TNF-alpha on axons. Moreover, pattern of immune response is not specific in SVN and NSVN, including vasculitic neuropathy of paraneoplastic syndrome.
\end{abstract}

\begin{abstract}
Abbreviations: SVN systemic vasculitic neuropathy, NSVN non-systemic vasculitic neuropathy, PNS peripheral nervous system, EC endothelial cells, LC leucocytes

\section{Introduction}

Vasculitis of PNS occurs a wide variety of pathological conditions such as auto-immune disorders, paraneoplastic syndromes and para-infectious diseases. This condition is classified in systemic vasculitis (systemic vasculitic neuropathy, SVN) when inflammation of vasculature is present in other tissues and non-systemic (nonsystemic vasculitic neuropathy, NSVN) in isolated PNS localisation [1]. Histological hallmarks of both are represented by thrombosis of vasculature determining hypoxia and axonal loss in peripheral nerves. Generally, endothelial tissue is involved in immune response without any damage. Some peculiarities of PNS endothelium, in example the poor amount of tight junctions and the frequent pinocytosis fenomena along with immunologic characteristic features such as the over-expression of major histocompatibility complex II and the elevated lymphocyte adhesion capacity of PNS in presence of ICAM1 (Intercellular Adhesion Molecule 1) may explain this phenomenon $[2,3]$. Moreover, endothelium has an active role in maintaining
\end{abstract}

the pro and anti-coagulation imbalance, while interleukins and TNF-alpha are known to have a clear pro-coagulant effect [4]. The endothelium itself produces cytokines that induce modification of adhesion molecules expression, thus promoting leucocytes adhesion and diapedesis. Nevertheless, the immunological mechanism causing the endothelium damage in vasculitis is still unclear, since generally the active role of endothelium in immune processes occurs without its damage. We examined the clinical and histological reports of seventy-five patients affected from vasculitis in order to differentiate the two forms of SVN and NSVN neuropathy. We also performed an immunohistochemical study on sural nerve of the same patients using antibodies directed to TNF-alpha, to interferon gamma (INF-gamma),

Correspondence to: Giovanni Antioco Putzu, MD, PhD, Casa di Cura Polispecialistica Santelena, Viale Marconi, 160, 09045 Quartu S.Elena, Cagliari, Italy, Tel: +390708605541, Fax: +39070837391, E-mail: puzzugio@gmail.com

Key words: systemic vasculitic neuropathy, non-systemic vasculitic neuropathy, cytokines, immune response, paraneoplastic syndrome

Received: December 02, 2016; Accepted: December 21, 2016; Published: December 23, 2016 
Putzu GA (2016) Cytokines and immune response in vasculitis of peripheral nervous system: Tumor necrosis factor-alpha and pro-inflammatory cytokines in vaculitis of peripheral nervous system: A comparative immunohistochemical study on systemic and non-systemic vasculitic neuropathy

to IL-1beta, to IL-4 (TH2 immune response), to activated complement fraction $\mathrm{C} 5 \mathrm{~b} 9$, to cluster of differentiation 3 (CD3, all lymphocytes), to CD4 (T-Helper lymphocytes), to CD8 (cytotoxic lymphocytes), to CD21 (B-lymphocytes), to CD11 (leucocytes and ICAM-1), to CD68 (macrophages), and finally to Major Histocompatibility Complex II (MHC II).

Sixty patients (33 males and 27 females, age range $24-89$ years, mean age 63 years) were classified as systemic vasculitic neuropathy. Seven patients were affected by rheumatoid arthritis, four patients by essential cryoglobulinaemia type II, five patients by monoclonal gammopathy of undetermined significance (MGUS), seven patients by polyarteritis nodosa, four patients by Churg-Strauss, three patients by Sjögren disease, five patients by HIV and other para-infectious conditions, three patients by paraneoplastic syndrome. Twenty-two patients were affected by unclassified systemic vasculitis.

\section{Patients and methods}

Sural nerve fragments of patients with SVN and NSVN were fixed in $2.5 \%$ glutaraldehyde, post-fixed in $1 \%$ osmium tetroxide, embedded in Araldite, and stained with hematoxylin-phloxin- saffron (HPS) and parapenylene diamine (PPD). Frozen sections of sural nerve ( $5 \mu \mathrm{m}$-thick) of five patients with SVN and NSVN were processed by immunohistochemistry with a panel of antibodies raised against interferon- $\gamma$ (IFN- $\gamma$, Genzyme, Cambridge, USA), IL- $1 \beta$ and Il-4 (Genzyme, Cambridge, USA), tumor necrosis factor- $\alpha$ (TNF- $\alpha$, Genzyme, Cambridge, USA), attack membrane complex C5b-9 (Dako, Paris, France), macrophage differentiating antigen CD68, CD3 and CD11 (Dako, Paris, France) and intercellular adhesion molecule ICAM1 (Immunotech, Marseille, France). Stain was revealed by the means of avidin-biotin-peroxidase complex technique. Five sural nerves obtained from patients affected by non inflammatory neuropathies were used as control.

\section{Results}

\section{Clinical and neurophysiological results}

The principal clinical patterns in SVN is represented by mononeuritis multiplex (26 cases) and mixed motor sensory symmetrical polyneuropathy ( 25 cases). In contrast, there was no clearly predominant clinical pattern in NSVN (Table 1 and 2). Electromyographic results of SVN and NSVN are indicated in Table 3.

Table 1. Clinical presentation of neuropathy in SVN and NSVN.

\section{SVN}

Pure sensory neuropathy

Mononeuritis

Mononeuritis multiplex

Mixed sensitive and motor asymmetrical polyneuropathy

Mixed sensitive and motor symmetrical polyneuropathy

Pure motor polyneuropathy

NSVN

Pure sensory neuropathy

Mononeuritis

Mononeuritis multiplex

Mixed sensitive and motor asymmetrical polyneuropathy

Mixed sensitive and motor symmetrical polyneuropathy

Pure motor polyneuropathy

\begin{tabular}{|l|c|}
\hline & \\
\hline & 1 case \\
\hline & 1 case \\
\hline & 26 cases \\
\hline & 6 cases \\
\hline & 25 cases \\
\hline & \\
\hline & 1 case \\
\hline hy & 1 case \\
\hline & 2 cases \\
\hline & 3 cases \\
\hline
\end{tabular}

Table 2. Electromyographic findings in SVN and NSVN.

\section{SVN}

Axonal damage with amplitude decrease of MNAP and SNAP, MCV and SCV studies normal or slightly decreased

Mixed axonal and Myelinic (secondary demyelination)

NSVN

Axonal damage with amplitude decrease of MNAP and SNAP, MCV and SCV studies normal or slightly decreased

Pure Myelinic (with conduction block)

Mixed axonal and Myelinic (secondary demyelination)

\begin{tabular}{|c|c|}
\hline & 58 cases \\
\hline 2 cases \\
\hline 12 cases \\
\hline 1 case \\
\hline 2 cases \\
\hline
\end{tabular}

Table 3. Immunoreactivity in SVN and NSVN.

\begin{tabular}{|c|c|c|c|c|c|c|c|c|}
\hline $\mathbf{P Z}$ & Sjögren & NC1 & RA & Paraneo & MGUS & N.C2 & PAN & NC3 \\
\hline Infiamm & + & $+/-$ & ++ & ++ & + & + & ++ & $+/-$ \\
\hline $\begin{array}{l}\text { CD3 } \\
\text { T-L }\end{array}$ & + & $+/-$ & ++ & ++ & + & + & ++ & $+/-$ \\
\hline $\begin{array}{l}\text { CD4 } \\
\text { T- H }\end{array}$ & + & $+/-$ & ++ & + & + & + & ++ & $+/-$ \\
\hline $\begin{array}{l}\text { CD8 } \\
\text { T-C }\end{array}$ & $+/-$ & $+/-$ & + & + & $+/-$ & + & + & $+/-$ \\
\hline $\begin{array}{l}\text { CD21 } \\
\text { L-B }\end{array}$ & - & - & - & - & - & - & + & + \\
\hline CD 11 & + & + & + & + & + & + & + & + \\
\hline CD68 & ++ & + & ++ & ++ & + & + & ++ & + \\
\hline C5b-9 & ++ & + & ++ & ++ & + & + & ++ & + \\
\hline IL-4 & - & - & - & - & - & - & $+/-$ & - \\
\hline INF- $\gamma$ & - & $+/-$ & ++ & + & + & + & + & - \\
\hline TNF- $\alpha$ & + & + & + & + & + & + & + & + \\
\hline MHC II & + & + & + & + & + & + & + & $+/-$ \\
\hline
\end{tabular}


Putzu GA (2016) Cytokines and immune response in vasculitis of peripheral nervous system: Tumor necrosis factor-alpha and pro-inflammatory cytokines in vaculitis of peripheral nervous system: A comparative immunohistochemical study on systemic and non-systemic vasculitic neuropathy

Table 4. Immnunolocalization of antibodies in sural nerve.

\begin{tabular}{|c|c|c|c|c|}
\hline Immune localization & Perineurial space & Blood vessel & Axons & Myelin \\
\hline CD3 T-L & $+/-$ & ++ & - & - \\
\hline CD4 T-H & + & ++ & - & - \\
\hline CD8 T-C & $+/-$ & $+/-$ & $+/-$ & - \\
\hline CD21 L-B & - & - & - & - \\
\hline CD 11 L+ICAM-1 & + & + & - & - \\
\hline CD68 & ++ & ++ & ++ & ++ \\
\hline C5b-9 & ++ & ++ & ++ & ++ \\
\hline IL-4 & - & - & - & - \\
\hline INF- $\gamma$ & + & ++ & - & - \\
\hline TNF- $\alpha$ & + & + & + & $+/-$ \\
\hline MHC II & + & + & + & + \\
\hline
\end{tabular}

Table 5. Principal adhesion molecules and their ligands.

\begin{tabular}{|l|c|c|}
\hline \multicolumn{1}{|c|}{ Adhesion molecule } & cells & \\
\hline Selectins & EC & sialoGP \\
\hline Selectin E & EC (CD62) & Selectin L \\
\hline Selectin P & LC & LC, EC (CD54) \\
\hline Selectin L & LC, EC & LFA-1, MAC-1 \\
\hline IG superfamily & LC & LFA-1 \\
\hline ICAM-1 & EC & LFA-3 \\
\hline ICAM-2 & EC, LC, Platelets (CD44) \\
\hline ICAM-3 & LC (CD29) & VLA-4 \\
\hline VCAM-1 & & MIP-1b \\
\hline HCAM & & \\
\hline Integrins & LC (CD11a, CD18) & LC \\
\hline B-1 VLA-4 & LCD11b, CD18 \\
\hline B-2 LFA-1 & \\
\hline B-2 MAC-1 & & ICAM-1 \\
\hline
\end{tabular}

The results of immunohistochemistry in SVN are summarized in Table 4. Immune localization in SVN and NSVN are included in Table 5. The main findings, similar in all classes of SVN including paraneoplastic syndrome, were characterized by the absence of immunoreactivity to IL-4 and CD21 antibodies that are specific for Th-2 immune response (antibody mediated immunity). Only patients affected by polyarteritis nodosa showed a weak immunoreactivity to IL-4 (data not shown). The same pattern of Th-1 immunity was observed in NSVN. CD68 (macrophages) and the activated complement fraction C5b9 were immunolocalized in axons and myelin whereas TNF-alpha was mainly localized on axons although small amounts were individuated on myelin as well (Figure 1 and 2). All antibodies of the panel, except IL-4 and CD 21 were isolated from the endoneurial space and blood vessels. In particular, an important immunoreactivity to INF-gamma and CD11/ICAM1 was found on vessels both in peri and endoneurial spaces (Figure 3 and 4). Immunoreactivity to CD8 (cytotoxic lymphocytes) antibodies was also present on axons. Immunoreactivity of cytoxic lymphocytes was more striking than this for CD4 (T-Helper lymphocytes) MHC II was strongly isolated from all patients both in vasculature and axons (Figure 5). Controls were all negative for the complete panel used in our series.

\section{Discussion}

To our knowledge, immunohistochemical findings on cytokines and immune mechanisms of vasculitis in paraneoplastic syndrome have not been published yet. In contrast, data on immunoistochemistry in systemic vasculitic neuropathies are available in the scientific literature although our series includes a greater number of patients in a comparative immunohistochemical study of SVN and NSVN [410]. There was no differential pattern between SVN and NSVN. This

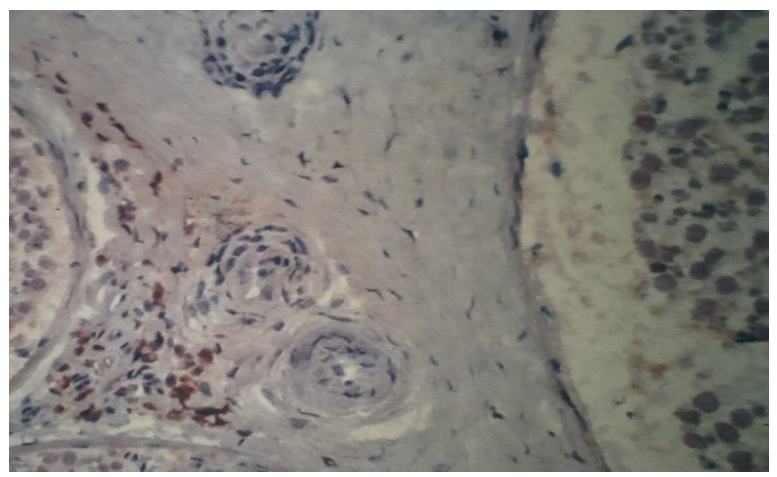

Figure 1. Immunohistochemistry with antibodies anti-CD68 (macrophages). Strong immunoreactivity in nerve fascicules and around small vessels in sural nerve of SVN, original magnification X100.

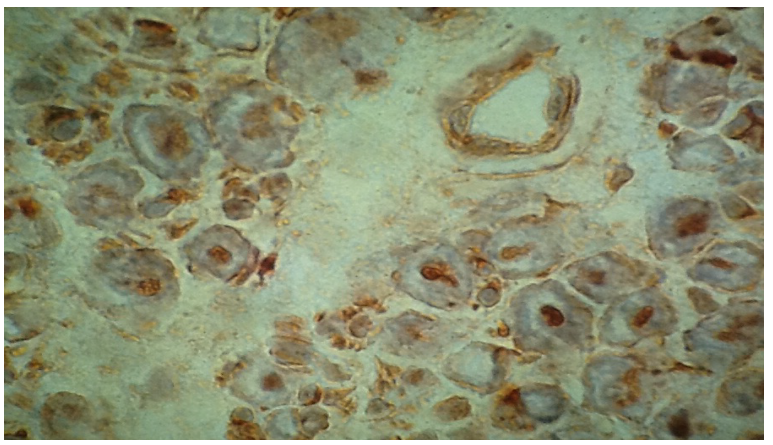

Figure 2. Immunohistochemistry with antibodies anti-TNF-alpha.

Immunoreactivity on axons, myelin and a small endoneurial vessel, original magnification $\mathrm{X} 400$. 
Putzu GA (2016) Cytokines and immune response in vasculitis of peripheral nervous system: Tumor necrosis factor-alpha and pro-inflammatory cytokines in vaculitis of peripheral nervous system: A comparative immunohistochemical study on systemic and non-systemic vasculitic neuropathy

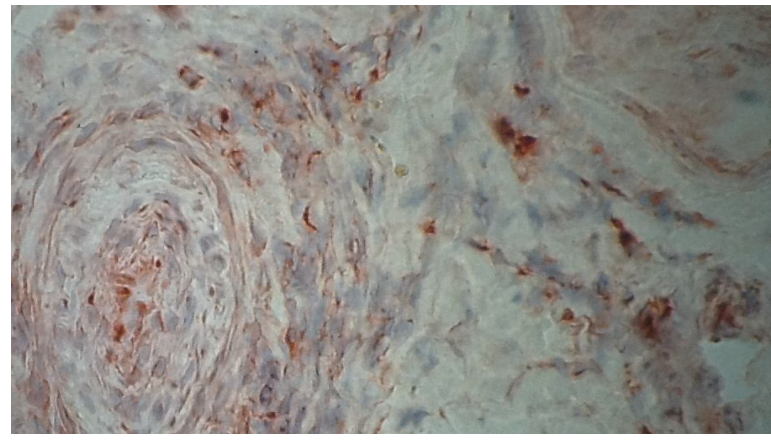

Figure 3. Immunohistochemistry with antibodies anti-INF-gamma. Immunoreactivity in a vessel with thrombosis, original magnification X400.

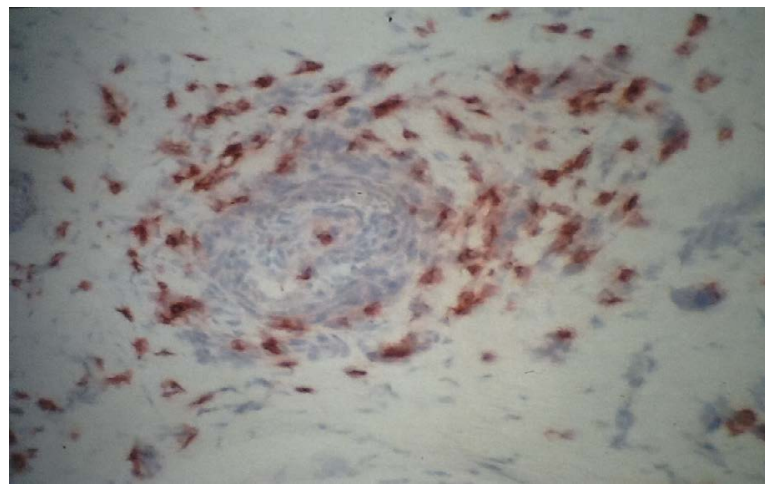

Figure 4. Immunohistochemistry with antibodies anti-ICAM-1/CD11.

Strong immunoreactivity in a vessel in sural nerve of SVN, original magnification X400.

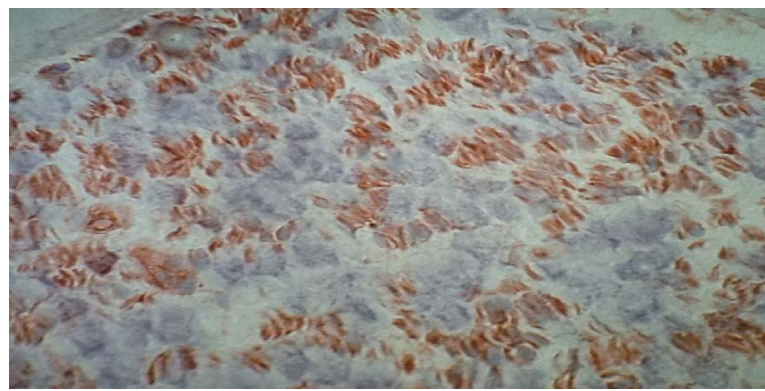

Figure 5. Immunohistochemistry with antibodies anti-MHC II.

Diffuse immunoreactivity in a nerve fascicules of SVN, original magnification X400.

finding has also never been previously reported. We demonstrated, by the means of double immunohistochemical technique, that TNF-alpha is responsible for myelin breakdown and axonal damage in GuillainBarré syndrome [11]. Macrophages along with $\mathrm{CD} 4$ positive cells stimulate the primary release of TNF-alpha. The secondary release of this cytokine, much more toxic than the primary release, is induced by INF-gamma and IL-2. These substances, that are TNF-alpha and INFgamma, were easily detected in our series, according to the previous published data and recent advances in the understanding of immune mechanisms. It is also known that INF-gamma production is stimulated by Il-28A, a powerful pro-inflammatory cytokine responsible for the amplification of the inflammatory process [12]. ICAM-1 was revealed in great amount in the vasculature along with CD11. This data is not surprising since CD11 is directed to integrins that act as a ligand to ICAM-1. The crucial role of ICAM in the acute phase of immune response is already known [13]. Nevertheless, in our series ICAM-1 was detected in most of the patients for months after the clinical onset of vaculitis. This finding clearly demonstrates that adhesion molecules have a long duration of expression in inflammatory processes. The same phenomenon was observed in Guillain-Barré syndrome [11].

The toxic effect of TNF-alpha is per se responsible for axonal degeneration in both SVN and NSVN although ischemia and thrombosis are a striking finding in vasculitis. Moreover, the procoagulant effect of TNF-alpha is the principal cause of thrombosis in vasculitis. According to the recent advances in other immune processes, we found a predominance of cytotoxic T-lymphocytes (CD8) in sural nerve of patients with respect to T-helper lymphocytes (CD4) in our study. This data suggests a more relevant effect of cytotoxicity on axons and blood vessels.

Clinically, SVN has a poor prognosis with a significant mortality rate [1]. In our series, one third of the patients affected by SVN were unclassified despite a frequent concomitant muscle biopsy that ameliorates the diagnostic accuracy. The treatment of SVN is based on the administration of high dosages of corticosteroids and immunosuppressant drugs, mainly cyclophosphamide. In case of SVN associated with cancer, corticosteroids and immunosuppressant drugs could worsen the prognosis of the disease by facilitating the progression of the tumour [14].

Promising advances in treatment of vasculitis in paraneoplastic syndrome may be provided by monoclonal antibody treatment of SVN in the future [15]. Our study further emphasizes the importance of Th-1 immune response in paraneoplastic neuropathy, according with the previous data of scientific literature $[16,17]$

\section{Acknowledgements}

We thank J.F Pellissier and D. Figarella-Branger (Marseille, France) with gratitude. We also thank N. Bianco (Marseille, France) for her worthy technical support.

\section{References}

1. Dyck PJ, Benstead TJ, Conn DL, Stevens JC, Windebank AJ, et al. (1987) Nonsystemic vasculitic neuropathy. Brain 110: 843-853. [Crossref]

2. Mitchell GW, Williams GS, Bosch EP, Hart MN (1991) Class II antigen expression in peripheral neuropathies. J Neurol Sci 102: 170-176. [Crossref]

3. Larsson P, Ulfhammer E, Karlsson L, Bokarewa M, Wåhlander K, et al. (2008) Effects of IL- $1 \beta$ and IL- 6 on tissue-type plasminogen activator expression in vascular endothelial cells. Thromb Res 123: 342-351. [Crossref]

4. Fenzi F, Rossi F, Rava M, Cavallaro T, Ferrari S, et al. (2006) Endothelial adhesion molecule expression is unaltered in the peripheral nerve from patients with AIDS and distal sensory neuropathy. J Neuroimmunol 178: 111-116. [Crossref]

5. Üçeyler N, Geng A, Reiners K, Toyka KV, Sommer C (2015) Non-systemic vasculitic neuropathy: single-center follow-up of 60 patients. J Neurol 262: 2092-2100. [Crossref]

6. Engelhardt A, Lörler H, Neundörfer B (1993) Immunohistochemical findings in vasculitic neuropathies. Acta Neurol Scand 87: 318-321. [Crossref]

7. Kurz M, Pischel H, Hartung HP, Kieseier BC (2005) Tumor necrosis factor-alphaconverting enzyme is expressed in inflammed peripheral nervous system. $J$ Peripher Nerv Syst 10: 311-318. [Crossref]

8. Lindenlaub T, Sommer C (2003) Cytokines in sural nerve biopsies from inflammatory and non-inflammatory neuropathies. Acta Neuropathol 105: 593-602. [Crossref]

9. Empl M, Renaud S, Erne B, Fuhr P, Straube A, et al. (2001) TNF-alpha expression in painful and nonpainful neuropathies. Neurology 56: 1371-1377. [Crossref]

10. Deprez M, Lübke U, Verlaet M, Debrus S, Delvenne P, et al. (2001) Detection of cytokines in human sural nerve biopsies: an immunohistochemical and molecular study. Acta Neuropathol 101: 393-404. [Crossref]

11. Putzu GA, Figarella-Branger D, Bouvier-Labit C, Liprandi A, Bianco N, et al. (2000) Immunohistochemical localization of cytokines, $\mathrm{C} 5 \mathrm{~b} 9$ and ICAM-1 in peripheral nerve 
Putzu GA (2016) Cytokines and immune response in vasculitis of peripheral nervous system: Tumor necrosis factor-alpha and pro-inflammatory cytokines in vaculitis of peripheral nervous system: A comparative immunohistochemical study on systemic and non-systemic vasculitic neuropathy

of Guillain-Barré. J Neurol Sci 174: 16-21. [Crossref]

12. Li B, Xie C, Lin X, Su SB (2014) Interleukin-28A promotes IFN- $\gamma$ production by peripheral blood mononuclear cells from patients with Behcet's disease. Cell Immunol 290: 116-119. [Crossref]

13. Schäfers M, Schmidt C, Vogel C, Toyka KV, Sommer C (2002) Tumour necrosis factor alpha (TNF) regulates the expression of ICAM-1 through TNF receptor 1 after chronic constriction injury of mouse sciatic nerve. Acta Neuropathol 104: 197-205. [Crossref]

14. Naddaf E, Dyck PJ (2015) Vasculitic Neuropathies. Curr Treat Options Neurol 17: 374. [Crossref]
15. Bramson C, Herrmann DN, Carey W, Keller D, Brown MT, et al. (2015) Exploring the role tanezumab as a novel treatment for the relief of pain. Pain Med 16: 1163-1176. [Crossref].

16. Tani T, Tanaka K, Idezuka J, Nishizawa M (2008) Regulatory T cells in paraneoplastic neurological syndromes. J Neuroimmunol 196: 166-169. [Crossref]

17. Antoine JC (2000) Immunological mechanisms in paraneoplastic peripheral neuropathy. Clin Rev Allergy Immunol 19: 61-72. [Crossref]

Copyright: (2016 Putzu GA. This is an open-access article distributed under the terms of the Creative Commons Attribution License, which permits unrestricted use, distribution, and reproduction in any medium, provided the original author and source are credited. 